\title{
Challenges for Life in the Local Universe
}

\author{
Paul A. Mason*t \\ New Mexico State University, Las Cruces, NM, USA \\ Picture Rocks Observatory and Astrobiology Research Center, Las Cruces, NM, USA \\ E-mail: pmason@nmsu.edu
}

\section{Peter L. Biermann}

Max Planck Institute for Radio Astronomy, Bonn, Germany

Karlsruhe Inst. für Tech. Karlsruhe, Germany

Univ. of Alabama, Tuscaloosa, AL, USA

Fachb. für Phys. \& Astron., Univ. Bonn, Germany

\begin{abstract}
We address some of the major astrophysical challenges facing present day life, including humans, in the local universe. A truly successful species must break free of the cycle of evolutionary development punctuated by mass extinctions. This must be accomplished before the end of the relatively short habitability lifetime of its planet and within a window between major mass extinctions. It is not inevitable that life will produce an apex species capable of understanding its vulnerability to extinction. Individuals must build a civilization - requiring hyper-social skills, and develop technology - requiring extraordinary mechanical skills. They must have the desire to understand the natural world and the universe well enough to anticipate, and even prevent, a variety of catastrophes. Ultimately, a space faring species is required; a quality not selected for naturally. Getting that far requires a great deal of luck. Getting beyond that, for humans and extraterrestrials alike, will require a sophisticated understanding of the physical universe and the accomplishment of tremendous feats of engineering.
\end{abstract}

In particular, a successful species must learn to leave its home planet to avoid the demise of its host star. Eventually in fact, the Galaxy itself will become inhospitable to complex life during the Milky Way collision with the Andromeda galaxy. Long before that they must find away to the understanding that their home planet requires protection from external threats, like asteroids, supervolcanoes, and supernova explosions; as well as from internal threats like plague and pestilence, for example the Covid-19 (corona virus) pandemic, as well as destruction by rogue elements and 'evil empires'. Most importantly, we argue for the need to avoid foreseeable catastrophe aided by science and reason. Natural selection has produced people capable of all manner of altruism or atrocity. Mass delusion is a cultural phenomenon. History shows that mass delusion, as a rule, existed throughout the pre-scientific world and persists today. This is despite the extraordinary effectiveness of science in improving people's lives. Finally, humans will face ethical challenges, if and when we encounter extraterrestrial life. Consider that much of the technological advancement of society today has been the result of the quest for competitive military advantage. We should expect the same from any technological exospecies. A major challenge for humans today is to learn to self-govern within the frame-work of evidenced based research. Research that is aimed at understanding and protecting the environment within which we have all evolved to survive.

The Golden Age of Cataclysmic Variables and Related Objects V (GOLDEN2019)

2-7 September 2019

Palermo, Italy 


\section{Introduction}

\subsection{The emergence of complex life}

When considering the emergence of life in the universe, it is useful to place qualifications on the nature of that life. For example, conditions that allow for the emergence of simple unicellular life-forms are significantly less strict than those allowing the development of complex multicellular life. The latter is capable of exponentially more complex behavior. Simple life undoubtably begets more complex life if the conditions are right. However, complex life likely requires the presence of a distinctively different set of environmental conditions than does simple life [1]. For just one major example: oxygen was deadly to early lifeforms, yet it is crucial now for today's voracious energy consuming animals. Some planets may produce single celled life, possibly many times, without making the leap to complex life. That is to say that multicellular and especially conscious life forms may be possible only under stricter (Earth-like or better) habitability conditions. Those conditions are possible on a much smaller subset of worlds. Time plays a critical role. Earth's transition to a nitrogen plus oxygen rich atmosphere allowed for high energy consuming animals, but only after billions of years. In other words, it is not sufficient to say this planet or that one is habitable. A more important question, and a more difficult one to answer is:

\section{What kind of life will a particular habitable planet support?}

Here we examine some of the challenges faced by life in the present day local universe. It is based largely on the vast literature from several generations of investigators in a variety of fields summarized in our review [2] as well as anecdotal insight into how the development of life is playing out on Earth.

Before life can emerge on a planet, the ingredients for life must be synthesized and concentrated to form planets in the first place. Inhomogeneity in the early universe resulted in the formation of clusters of galaxies. The presence of dark matter probably acted as a catalyst for galaxy formation. Galaxies provide the necessary conditions for the formation of stars. Stellar nucleosynthesis and the subsequent chemical enrichment, transforms a galaxy from an uninhabitable stellar system into a habitable one. The key factor is the concentration of the so-called CHNOPS set of elements.

The transition from simple to complex life on Earth took a great deal of time. In fact, it took most of Earth's history. First consider that simple life appeared early in Earth's history. Fossil evidence shows that life arose at most only a few hundred million years after Earth became fully formed [3], being not long after the crust cooled enough for surface water. It took far longer for multicellular species to develop, and billions of more years for the full blooming of life as we know it to arise. The frequency of global catastrophe played a significant role in evolution on Earth. First in setting the time for the emergence of the increasingly more complex life forms and then to set the clock on the race to evolve to a space faring species before the next mass extinction. But the need to protect life from the astrophysical environment begins long before the present-day stage of development.

\footnotetext{
*Speaker.

${ }^{\dagger}$ This work supported by PRO-ARC
} 
Darwinian natural selection and mutations allow for the rise and fall of species, however mass extinctions transmute life on Earth at the kingdom and phylum level. The surface of the Earth has been alternately dominated by fungi, plants, and animals; each contributing to a complex web-oflife. During a vast expanse of time, creatures of the biosphere existed completely at the mercy of the physical environment. Diversity developed, usually intermittently as niches closed, precipitating extinction, and then re-opened to others after mass extinction events. This diversity has resulted in life forms of all types, whose lineages have all already survived many mass extinctions. To emphasize the point, note that every living creature on Earth today has ancestors that survived every mass extinction in Earth's history since the last universal common ancestor (LUCA) [4].

Hominins arose only 5-7 million years ago, well after the most recent major mass extinction, 65 million years ago. Ancestors of present day humans had to overcome natural predators and then had to learn to deal with each other, and members of related species, to immigrate out of Africa and across the planet. They have taken many steps towards building a protective technological civilization during the last 70,000 years. They had to develop agriculture, tools, and social skills in order to survive in a competitive world. Ultimately, science allowed for the rise of the technological civilization we enjoy today. To illustrate this point, note that the great age of modern science originated with Newton in the 17th century amid the Great Plague of London. The bubonic plague spreads through bacteria carried by fleas living on common black rats. It ravaged Europe, off and on, for at least one thousand years. Achievement during plague outbreaks was not limited to science. Shakespeare, whose writing was famously influenced by his personal state of mind, wrote many works during outbreaks. His play company even cancelled performances much of the time between 1603 and 1613 due to legal restrictions during epidemics. Today, the disease is eradicated in Europe and is in retreat throughout the world despite the fact that there is no known cure or proven vaccine. Simple knowledge of the cause led to effective prevention in this case. In the last one-hundred years the germ theory for diseases has identified many species responsible for epidemics plaguing the world's emerging cities across history. This resulted in a human population explosion. In order to become a space-faring planet-protecting species, large populations in crowded spaces are likely to develop first. This necessitates the development of societies, as well as science to maintain them.

We suggest that technological civilizations have only relatively recent emerged in the local universe due to the enormous time likely needed to evolve them amidst an unforgiving universe. The habitability of a planet for complex life as we know it depends on properties of the host galaxy [2]. In particular its merger and star formation histories. Further, we suggest that challenges for technological civilizations are far from over. Some of these challenges may be overcome by engineering efforts. For example, any technological society will need to quickly defend its civilization against otherwise inevitable asteroid impacts. Some harmful astrophysical factors, like Gamma-Ray Bursts (GRBs), maybe unavoidable for some planets. Unquestionably, for any society to succeed beyond the habitable lifetime of their home world, a high degree of technology aided by considerable luck must be achieved.

\subsection{Ingredients of life}

The elements used by life on Earth were not available until long after the Big-Bang. Nucleosynthesis during the Big-Bang resulted only in the production of helium and small amounts of other light elements, see the discussion in Mason and Biermann (2018) [2]. For much of the 
history of the universe, the ingredients for life were present only in small quantities or more precisely elements were likely not sufficiently concentrated to facilitate life. Dark matter is poorly understood, however what seems to be clear is that galaxies, and life itself, exist today because of an ancient lumpiness of dark matter in the universe. When we look at quasars, we see accreting supermassive black holes, some of which are seen frenetically growing less than a billion years after the Big-Bang; we are seeing the beginning effects of a messy process that may result in a habitable galaxy or it may not. The richest clusters of galaxies are lit up by active galaxies and lie at nexus points of dark matter filaments stretching across the universe. The first black holes at the centers of the first galaxies might have been produced by the collapse of hydrogen and helium gas clouds devoid of the heavier nuclei required for potentially life-bearing planets. It could also just be the agglomeration of massive stars, forming a super-massive star, which is unstable, and makes directly a million solar mass black hole [5]. There are also other options, since we do not know what dark matter is. The collapse of smaller concentrations, less than about $100 \mathrm{M}_{\odot}$ of baryonic matter formed the first stars. The production of metals, elements beyond hydrogen and helium on the periodic table, is the result of the interplay between dark and visible matter.

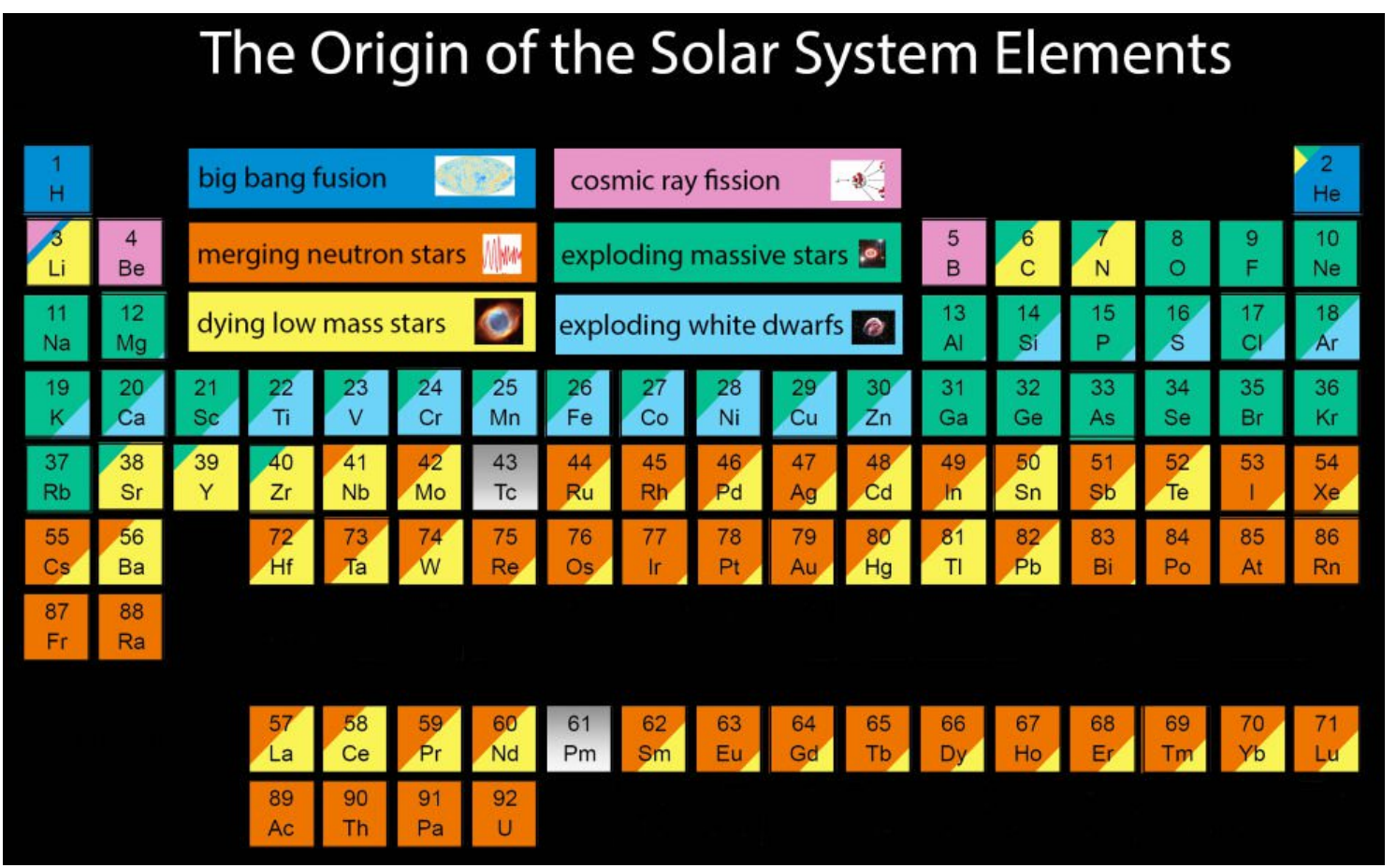

Figure 1: The periodic table of the origin of the elements. The elements originate from a variety of sources, see legend, and the time-scale for their production varies significantly depending of the type of source. Credit: Jennifer Johnson (2019) [6].

Concentrations of silicates, iron, carbon, water and so on, in molecular clouds will make their way into protoplanetary disks and eventually into planets. It took the first generations of stars to fuse hydrogen and helium into carbon, nitrogen, and oxygen, to build life. It took quite a bit longer to have significant concentrations of iron and silicates, to build planets. In Figure 1, a periodic table listing the origin of each element is shown. We see that the elements needed to make rocky planets, 
$\mathrm{Fe}, \mathrm{Ni}, \mathrm{Si}, \mathrm{Mg}$, and so on, are formed mainly by short lived high-mass stars. The more massive of the many, longer lived, low-mass stars supply $\mathrm{C}, \mathrm{N}, \mathrm{O}$ and $\mathrm{P}$, which are the constituents of organic molecules. The abundance of all of these elements relative to hydrogen steadily increases over the lifetime of a galaxy. The chemical enrichment of the Milky Way over time is quickly becoming quite well understood generally, due especially to surveys like the Sloan Digital Sky Survey and LAMOST spectroscopic surveys to map the present day chemical architecture of the Galaxy. In addition, the Gaia space observatory is determining the distance, using parallax, to the billion brightest stars visible from Earth and proper motions of the closest of these. As of this writing, measurements are being made that when combined will reveal the 3-D motions and the chemical composition of as many stars in the Milky Way as there are seconds in a human lifetime. These data will be highly valuable to Galactic habitability studies.

The details for how the ingredients for life facilitated the origin of life remains one of the most important questions in science today. During the process of star formation, exposure of the molecules to ultraviolet radiation provides for a rich chemistry that results in the molecular building blocks of life. Organic molecules must overcome significant hurdles to become life and time is a key ingredient. This enrichment may occur at various points along, potentially, a wide variety of paths from molecular cloud to planetary surface. The environment those molecules experience directly influences their potential to participate in prehistoric chemistry and hence in life itself. The surfaces of planets (or moons) in the oceans and on land, provide the concentrated ingredients for life as we know it. Clearly, this elemental concentration is universal in nature as galaxies, stars, and planetary systems undoubtably form and develop across the universe. However, rationally investigating the impact of surface radiation on planets requires overcoming the natural Geocentric bias resulting from current placid conditions on Earth. For example, it is possible that some circumbinary planets may posses 'better than Earth' habitability, based on a reduction of stellar XUV and particle flux in the habitable zones of some binaries [7, 8, 9].

The star formation rate in the Milky Way is now considerably less than it was during its early history. Star formation began near the center of the Galaxy and stars soon populated the halo. The bulge and bar formed stars relatively quickly while the Galactic disk slowly formed stars in the so called thick disk. Over time, the gas and the dust (expelled from dying stars) in the disk collapsed into what is now the thin disk, further concentrating the elements for life. We suggest the vast majority of stars formed in the halo, the bulge, and even the thick disk are too metal poor to allow the development of wet rocky planets with their habitable zones [2]. While this simple picture is largely correct, star formation in most galaxies occasionally has huge spikes, called a starburst; during such periods of time, life especially complex surface life is not viable. So our galaxy apparently never had a significant starburst period, at least not in the last 4.5 billion years. But it almost certainly will have such a period in about 4 - 5 billion years during the collision between Milky Way and the Andromeda Galaxy! [10, 11]

In order to allow Earth-like living conditions, generations of stars produced the necessary elements allowing for a sustained planetary habitability environment. The concentration and abundance of a variety of elements are needed for the development of complex life. Not only do galactic disks allow for the concentration of the CHNOPS elements for life and the planet building elements $\mathrm{Si}$ and $\mathrm{Fe}$, they also concentrate radioactive elements like Al, Th, U. The presence of very long half-life radioactive elements $\mathrm{Th}$ and $\mathrm{U}$ on Earth have allowed for a prolonged period of geologic 
activity. This is because radioactive elements have helped maintain high Earth core and mantle temperatures. Radioactive isotopes of certain elements, see Figure 1 are produced in neutron star mergers. The fact that these events are rare means that their nucleosynthesis products may be rather non-uniformly distributed. One or two local neutron star mergers are probably responsible for much of the Th and U content of Earth. Having too little radioactivity results in a briefer geological activity lifetime; while too much radioactivity likely results in more frequent volcanic eruptions, like the one that caused the End Permian extinction, only 250 million years ago, where $96 \%$ of all species on Earth were lost due to a prolonged period of hyperactive volcanism. Planets with too high a radioactive content may take too long to settle into a life sustaining environment for complex life.

It has long been predicted that in order for the rare elements beyond iron to be formed, high fluxes of neutrons must be involved [12]. Models for element production during neutron star mergers suggest that they are a probable r-process site [13], since there should be plenty of high energy neutron-ion interactions. The discovery of the neutron-star merger GW170817 by gravitational wave detectors [14] and observations of the the optical/infrared transient, called a kilonova that became visible days after the merger confirmed the connection between neutron star mergers and the production of Strontium [15], the lightest of the r-process elements that are fused in the presence of lots of neutrons. While some details of Figure 1 remain to be worked out, it is clear that binary neutron star mergers play an important role in constraining the habitability for complex life in the universe.

\section{Energy for life}

Life as we know it exists due to local energy disequilibrium. All organisms must make a living by the extraction of energy from their environments. Schrödinger [16] postulated an 'aperiodic crystal' containing heritable genetic information before the discovery of DNA. He also describes a living organism as one that maintains low entropy. The entropy decrease inside an organism is more than compensated by the entropy increase outside the organism, by heat expelled into the environment. The second law is obeyed, and life maintains a highly ordered state, In order to operate on Earth, (Gibbs) free energy is needed and in our case it is provided by the Sun. Chemical and energy disequilibrium provide a means for energy extraction and entropy reduction at the molecular and organism level. Liquid water is required in order for all life to operate, so there exists a temperature range based on the liquid water phase, within which life must remain.

Too much energy is destructive to life forms. Particle fluxes from radioactive surface rocks and cosmic rays are destructive at extreme levels. Cosmic rays at fluxes that are much higher than present day Earth levels are likely harmful to surface life and may have caused mass extinctions. On the other hand radiation levels that are only somewhat higher than present day Earth might have beneficial effects. Cosmic ray interaction with DNA molecules along with radioactive decay products are a source of genetic mutations. General principles thus suggest that there is an optimal range for radiation allowing for sufficient mutation without compromising too many organisms. The radiation level on Earth has gradually decreased from very high levels to present day low levels due to the decrease in the star-formation rate within the Milky Way over the history of the Earth [2], among other factors. 
Life also needs energy that may be supplied by:

1) the host star(s)

2) local geophysical or geochemical processes

3) external planetary system and astrophysical environmental impact.

On Earth, we are potentially biased in considering primarily the influence of i) the host star on the presence and the subsequent evolution of life on Earth. That bias is naturally transferred to the study of exoplanet habitability. So here, we take the position that the world around us provides sufficient evidence to suggest that the development of an exospecies capable of self preservation, against planetary catastrophe, is a significant challenge. This idea was expressed as one of the terms in the Drake equation factor, $f_{i}$, which is the probability that simple life will result in intelligent life. We suggest further, that the development of a technological civilization capable of self-preservation may be quite small. A planet's position in the habitable zone is hidden in this term of the Drake Equation. However, it is important in limiting the habitable planetary lifetime and in determining the potential total bio-production of a planet.

For example, consider that Earth is near the inner edge of the current habitable zone of the Sun. If the Earth were farther from the Sun, then it would have received less solar insolation, according to the inverse square law, for the entirety of Earth's past. Even assuming, in this thought experiment, that life might begin at the same time on this imaginary Earth currently in the middle of the habitable zone. That hypothetical planet would remain in the habitable zone a bit longer than the real Earth, because the habitable zone will gradually move outward as the Sun increases luminosity on its way to becoming a red giant. However, that imaginary Earth would receive about $50 \%$ less energy integrated over its lifetime. Significantly less energy from the Sun means, that among other things, it would have taken correspondingly longer for the Great Oxygenation Event to occur, whereby on Earth, photosynthetic bacteria changed the atmosphere and paved the way for animals. A biosphere can only change the atmosphere if it is consuming an enormous amount of energy.

It is often taken for granted that our existence was an inevitable consequence of what was before. It has taken a significant fraction of the habitable lifetime of the Earth for humans to become capable of self preservation, meaning capable of avoiding extinction. Life originated roughly 3.5 billion years ago and the Earth will no longer be in the habitable zone of the Sun in about 1.5 billion years. That means that life on Earth took $70 \%$ of Earth's habitability life-time to become (almost) capable of self-preservation. If this time-scale is typical, then it is sensible that a reduction of available energy for life would lengthen the time needed to life to meet this challenge. There is likely even a energy threshold below which life is incapable of global influence and much less capable of global protection.

Once produced, the energy content of $\mathrm{O}_{2}$ allows for high energy consuming species. Without sufficient energy, life is not capable of environmental transformation, critical to the development of complex life on Earth. On the other hand, if the Earth were even just a few percent closer to the Sun, then no matter how rapidly complex life would have developed, it would have ended by now. We speculate that the extremely high oxygen consumption of the human-like brain is only possible on the surface of planet with a high energy consuming biosphere near the inner edge of 
the habitable zone, just like Earth.

Certainly, for life to progress beyond the limitations of even it own planetary system environment, life (maybe our decedents) must meet some major challenges. Life has to develop a protection from, or at least a resilience against, extrinsic catastrophes such as asteroid collisions, nearby supernovae ( $\mathrm{SNe}$ ) and GRBs as well as intrinsic catastrophes such as nuclear destruction, or pestilence like the 1918 flu or the current coronavirus pandemic, considering that some governments speculate about herd immunity implying the death of a significant percentage of the entire population. Despite these setbacks, the process of establishing sustainability for life is well underway; consider that the lineage of every living creature on Earth has survived countless mass extinctions. Before going on to discuss potential future challenges, we review some of the external dangers faced by life.

\section{The universe is generally dangerous to life}

High energy radiation, both electromagnetic; ultraviolet (UV), X-Rays, and gamma-rays as well as cosmic rays (meaning particles) contribute to the environment experienced by life. The environment experienced by the molecular building blocks of life, at various stages is key. For example, the composition of planets and even entire planetary systems depends on details concerning the condensation of grains in the protoplanetary disk and on the impact of subsequent radiation. UV light from the newly forming star removes ice from grains located in the inner part of the protoplanetary disk and thus produces the so called snow-line, beyond which icy planetesimals may exist. Additional radiation, that is radiation from sources other than that from the parent star, may have a significant adverse impact on the planetary formation process itself. UV radiation from nearby O-type stars formed within the same cluster or worse, a close encounter with an OB association of a dozen or so hot UV intense stars during the time of planet formation, around an otherwise normal solar-type star, might not allow for the formation of wet rocky planets. If the nascent Earth had experienced much higher radiation levels, it would have had the effect of pushing the snowline far out in the protoplanetary disk or it could have even completely removed all water from the disk. This has obvious implications for the subsequent habitability for a planet formed from such a disk. A planet might be the right distance from its host star, but be bone dry. It makes no sense to consider the classical concept of a liquid water habitable zone in this case.

An Earth-mass planet may form dry in the habitable zone of a solar-type star, but could receive water later. Earth may have received much of its water from comet impacts well after its initial formation. If the snow-line is moved further out in a planetary system due to an intense period of cosmic ray bombardment, during the epoch of planetary formation, then giant planets would not be formed as a rule in desiccated regions of the protoplanetary disk. The fact that the snow-line of the Solar System is in the asteroid belt allowed ice to form beyond into cores which became the giant planets. Ice formation appears to be an important part of planetary formation in the Solar System case at least.

Stars and their putative planets in globular clusters are formed in the presence of many other stars forming during and around the epoch of protoplanetary disk formation and evolution into planets. The intense interstellar radiation and particle environment within which these planets form may inhibit water delivery to habitable zone planets because giant planets cannot form, as ice, a giant 
planet formation catalyst, and thus giant planets are not available to scatter comets to provide water delivery at early times, or, in fact, to protect life on a habitable zone planet by deflecting, capturing, or absorbing asteroids and comets at later (more critical) times in bio-development. Evidence exists that giant planet formation is strongly inhibited in regions of high radiation although very low metallicity is also a contributing and confounding factor. For example, the globular cluster 47 Tuc was observed using HST in an attempt to observe transits involving giant planets; a strong upper limit on the frequency of such planets was obtained as not a single planet was detected [17]. Earth-like (wet) planets may not be nearly as common as Sun-like stars.

\section{Astrophysical threats to life}

So what kinds of threats might life as we know it (or otherwise) face? These may be broadly grouped into three classes. Firstly exploding stars (supernovae) including the sometimes associated GRBs are extremely important. Supernovae are central to all aspects of life. Secondly accreting black holes, especially supermassive galactic center black holes, place strong limits on life. And thirdly, merging galaxies and the merging of their supermassive black holes may sterilize vast regions of otherwise habitable space. The astrophysical sources described in this section are considered to be threats particularly to complex life on surfaces of Earth-like planets. Microbial life or complex ocean life is expected to be more resilient to exposure to high levels of surface radiation. Some of the astrophysical threats to life as we know it on the surfaces of planets are discussed in this section. We focus on some of the challenges facing life. The development of complex life in the universe took a long time and is probably relatively recent in the universe. Mounting evidence, suggests that planetary habitability for complex life is fragile. Using nearby examples, we suggest that much the present day universe in inhospitable for life.

\subsection{Supernovae are central to life}

Supernovae ( $\mathrm{SNe}$ ) of all types play a central role for life in the Milky Way. A multi-wavelength view of the Crab Nebula is shown in Figure 2. Elemental enrichment of the ISM is the first step. The first generation of planets formed in a cluster might be sterilized by the removal of its atmosphere if too many $\mathrm{SNe}$ occur too near the new planet. The delivery of fresh radioactive material provided by $\mathrm{SNe}$ is a benefit, balanced by atmospheric impact. A nearby $\mathrm{SN}$ and/or being in the beam of a GRB is a significant threat to surface life.

Here we emphasize a key role for SN in the protection of life on planets in the thin disks of spiral galaxies, especially the Milky Way. The remnants of SNe currently populate the Galactic disk and are the sight of particle acceleration, $\mathrm{SNe}$ are the source of most of the galactic cosmic rays, meaning particle flux from Galactic sources. From the perpendicular (to the disk) flux of these high velocity particles combined with the differential rotation of the disk, a Galactic disk wind is established [18], see our review for an in depth discussion [2]. It is a Galactic magnetized wind that acts physically very much like the stellar magnetized wind. The magnetized stellar wind acts to deflect incoming galactic cosmic rays with energies below about $300 \mathrm{MeV}$. The galactic disk wind deflects incoming extragalactic cosmic rays, except for the Ultra High Energy Cosmic Rays (UHECRs). Without the protection of our Galactic disk wind, Earth would be subjected to an increased flux of very high energy radiation from a relatively nearby extragalactic source, for 


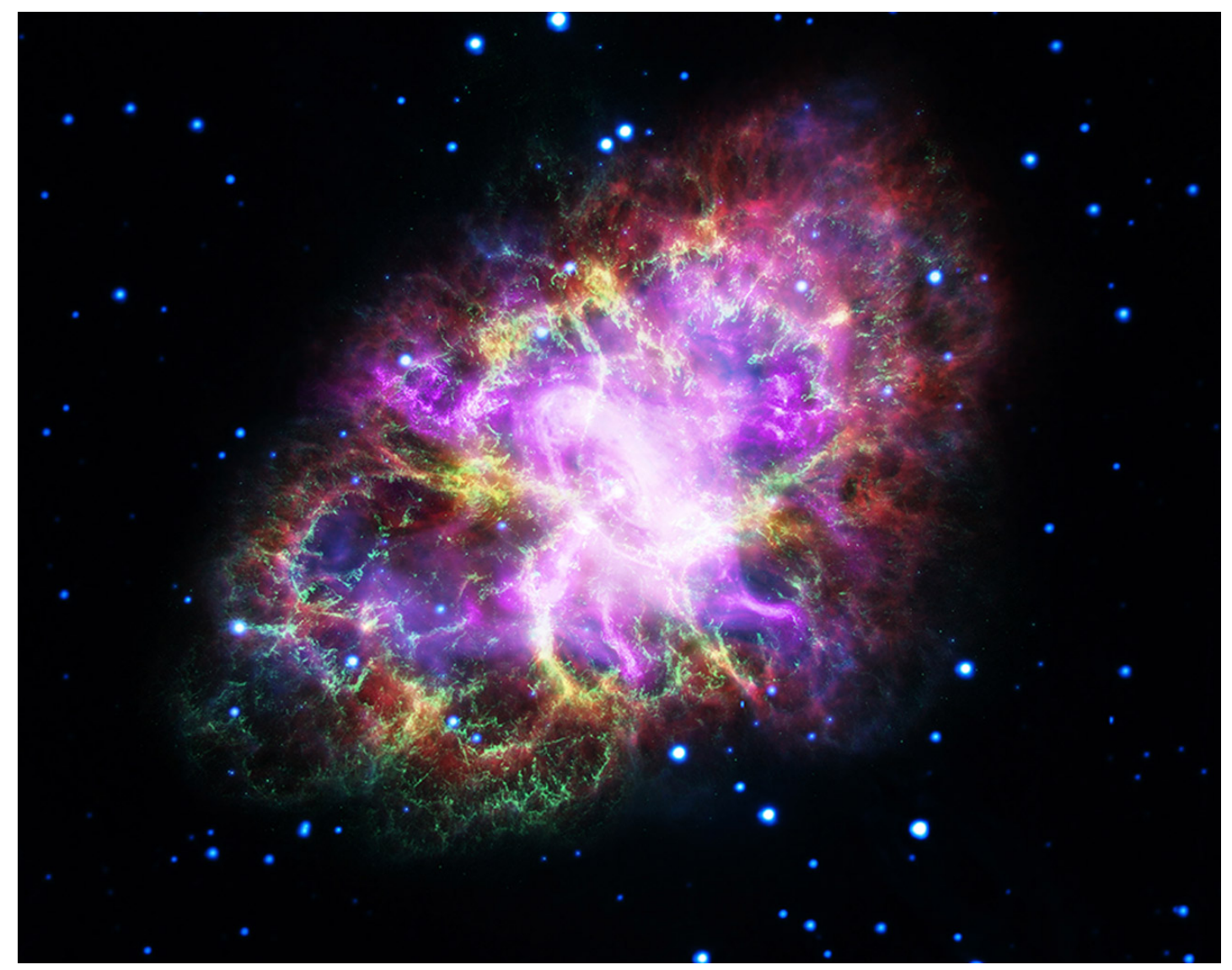

Figure 2: The Crab Nebula supernova remnant. A composite image from five observatories. For a short time after this supernova occurred in July of 1054, it was visible during the day. Supernovae are central to life. They fuse together the elements, especially carbon that life is based on, and then supernovae distribute the new elements into the interstellar medium. Besides the fusion of essential elements supernovae are connected to complex life in several possibly surprising ways. Supernovae remnants are the main site for the acceleration of Galactic cosmic rays. This particle flux is responsible for mutations. In addition, supernova explosions aided by differential rotation of the disk, produce a galactic wind flowing perpendicular from the galactic disk. This magnetized wind is akin to the solar magnetized wind. The solar wind supplies the heliosphere with particles that stop galactic cosmic rays with $\mathrm{E}<300 \mathrm{MeV}$ largely originating from supernovae remnants, from impacting life on Earth. Credits: VLA (radio) in red; Spitzer Space Telescope (infrared) in yellow; Hubble Space Telescope (visible) in green; XMM-Newton (ultraviolet) in blue; and Chandra X-ray Observatory (X-ray) in purple.

example an accreting black hole in a neighboring galaxy. It is very likely that a planet needs the protection of a galactic disk wind to maintain livable surface conditions at some point during its biological lifetime.

\subsection{Accreting black holes and merging black holes are dangerous}

When two black holes merge, their respective jets swing through a huge solid angle, see Figure 1 in Kronberg et al. [19] and its recent discussion in Biermann et al. (2018) [20], due to angular 
momentum conservation. Consider that orbital angular momentum of the black hole binary may greatly exceed the angular momentum of the individual black holes. In addition, relativistic jets are directed along the black hole spin axis. So there is often a completely different jet direction before the merger compared to after the merger. This is called a black hole spin-flip. See the general relativistic calculations of Gergely and Biermann [21]. Biermann et al. (2018) [20] suggest that the energies for the observed clean-out imply that the jets have to run at their respective Eddington limit [20]. They discuss the source 41.9+58 in M82 and argue that it is a binary black hole merger, with a large spin-flip angle. That implies that every planet in that precessional cone will experience the jet briefly. The jet solid angle divided by the swing solid angle, for this observed example, very roughly is $\frac{10^{-2}}{\pi}$, so of order $10^{-2.5}$. One can estimate the total swing time scale to be of order 100 years, so in other words, every planet within that swing solid angle would experience such a powerful jet for a few months. As all of this has to happen in an young stellar cluster, like a dense version of perhaps the Orion cluster. The nearby galaxy M82 shows many such young stellar clusters, as do many other starburst galaxies. One can make a corresponding argument for the powerful jets of super-massive black hole mergers, when the swing time scale could be millions of years, and so the jet exposure time would be thousands of years.

Whether merging stellar mass black holes contribute to any measurable effects on the Earth through their spin/jet-flips is an open question. However, Biermann et al. [20] estimates that events as seen in M82 happen every couple of thousand of years in such a starburst. That implies that the sum of the conical regions affected could reach all parts of the inner galaxy in a few million years, which is about the same time as the starburst lasts. So, as soon as we have a starburst in some small region, that region will be hit by two effects, the massive star $\mathrm{SNe}$, their radiation and cosmic rays, as well as by the spin-flipping of the jets. We can consider what temporal pattern such a model would follow. If the Earth gets hit more than once at all, it would be an accelerating pattern, with a third pass coming faster than the second pass, and a possible fourth pass yet faster. Many passes would seem unlikely, since the conal pattern gets narrower as well. A mass extinction on Earth due to the merger of a binary supermassive black hole in a nearby external galaxy may be detectable in mass extinction data. The effects on habitability may extend across the host cluster and the extent of the effects depend mainly on the mass of the SMBH. On the other hand, we caution that survivors bias might lead us to underestimate the severity of this kind of astrophysical catastrophe and therefore overestimate the likelihood of complex life.

\subsection{Merging galaxies and their supermassive black holes are threats}

Merging black holes, both stellar and supermassive, are likely to be the main source of UHECRs, high-energy gamma rays, and neutrinos. The effect of a black hole merger/GRB model in our Galaxy is equivalent to a black hole merger of order $10^{6}$ more mass at a distance $10^{3}$ more distant. So there should be concern for distances up to up to about $7 \mathrm{Mpc}$, clearly encompassing both the starburst galaxy M82 as well as Cen A, but not quite to M87 at the heart of the Virgo cluster, see Figure 3 showing the Virgo Supercluster and its central galaxies. The most recent supermassive black hole merger in M87 corresponded to a mass much higher than that in Cen A, so again it would be in the detrimental range, if we assume the Eddington limit in all cases for the spin-flip period, as seems evident in M82. Table 2 in Gergely et al. (2009) [21] gives the various time scales. In Gopal-Krishna et al. [22] there is a discussion of the time scales in Cen A. Of course that all 


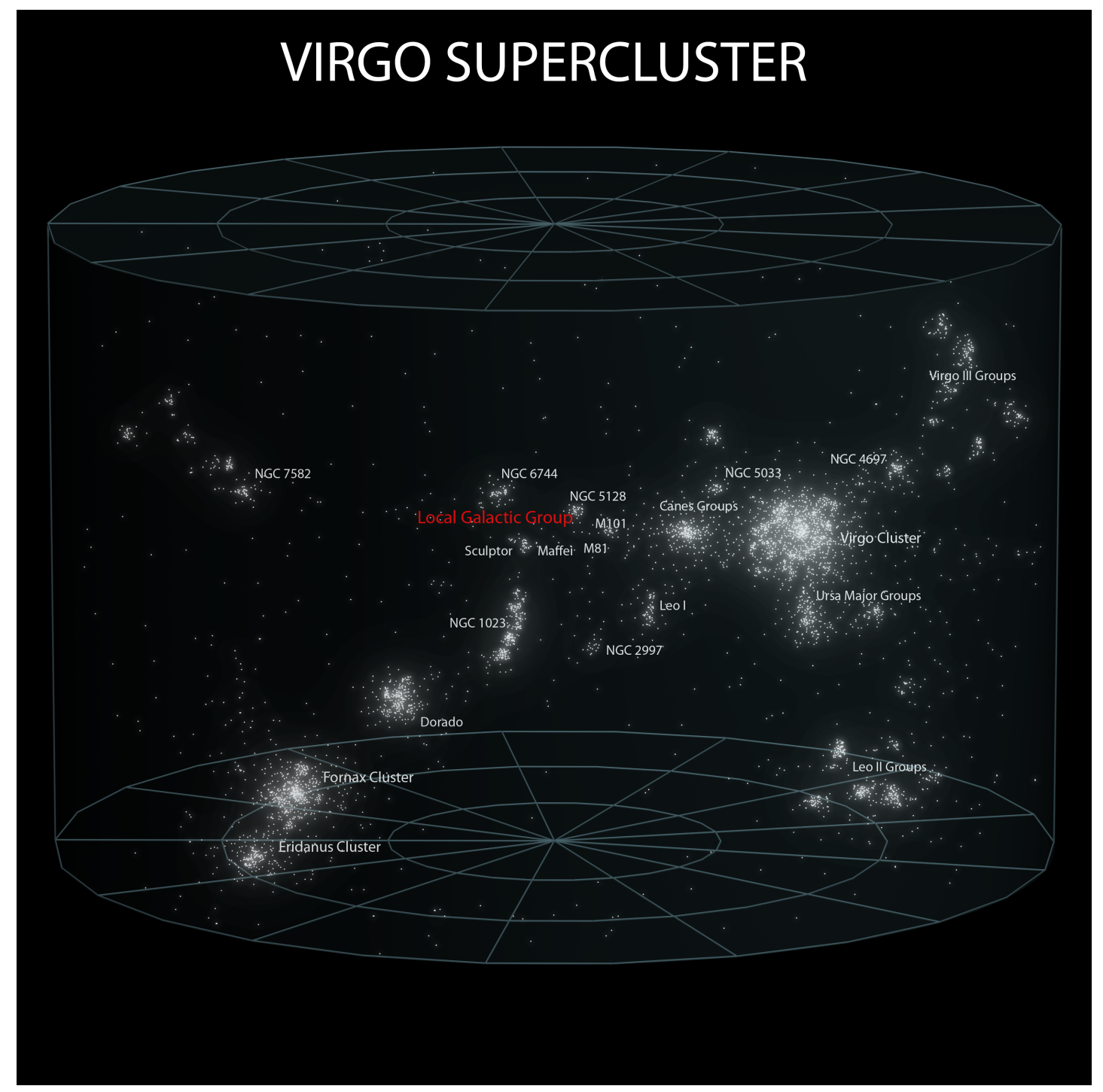

Figure 3: Map of the Virgo Supercluster of galaxies. The Local Group, in the center of the plot, containing the Milky and Andromeda galaxies, is in the low density suburbs of the Virgo Supercluster. Credit: Andrew Z. Colvin.

was a while ago, but close enough in time that in both cases (Cen A and M87) you can still see the old radio structures, at $90 \mathrm{~cm}$, in M87 as shown in Figure 4 [23]. It may be too speculative, but the overall image here of M87 in Figure 4, suggests a spin-flip angle of nearly 180 degrees, which is only possible if the two black holes merging are of very similar mass, as shown by the equations in Gergely \& Biermann [21]. That would have been maximally violent, if true.

To summarize, our existence is owed to the fact that we do not reside in, or even near, an active galaxy. The Milky Way has remained habitable, in this sense, due to a low frequency of major mergers. This is not to say that the Milky Way shows no evidence of central activity. For example, Figure 5 shows the high energy gamma-ray emission associated with particles trapped in the Fermi bubbles, which are located above and below the Galactic center. Dense galaxy clusters 


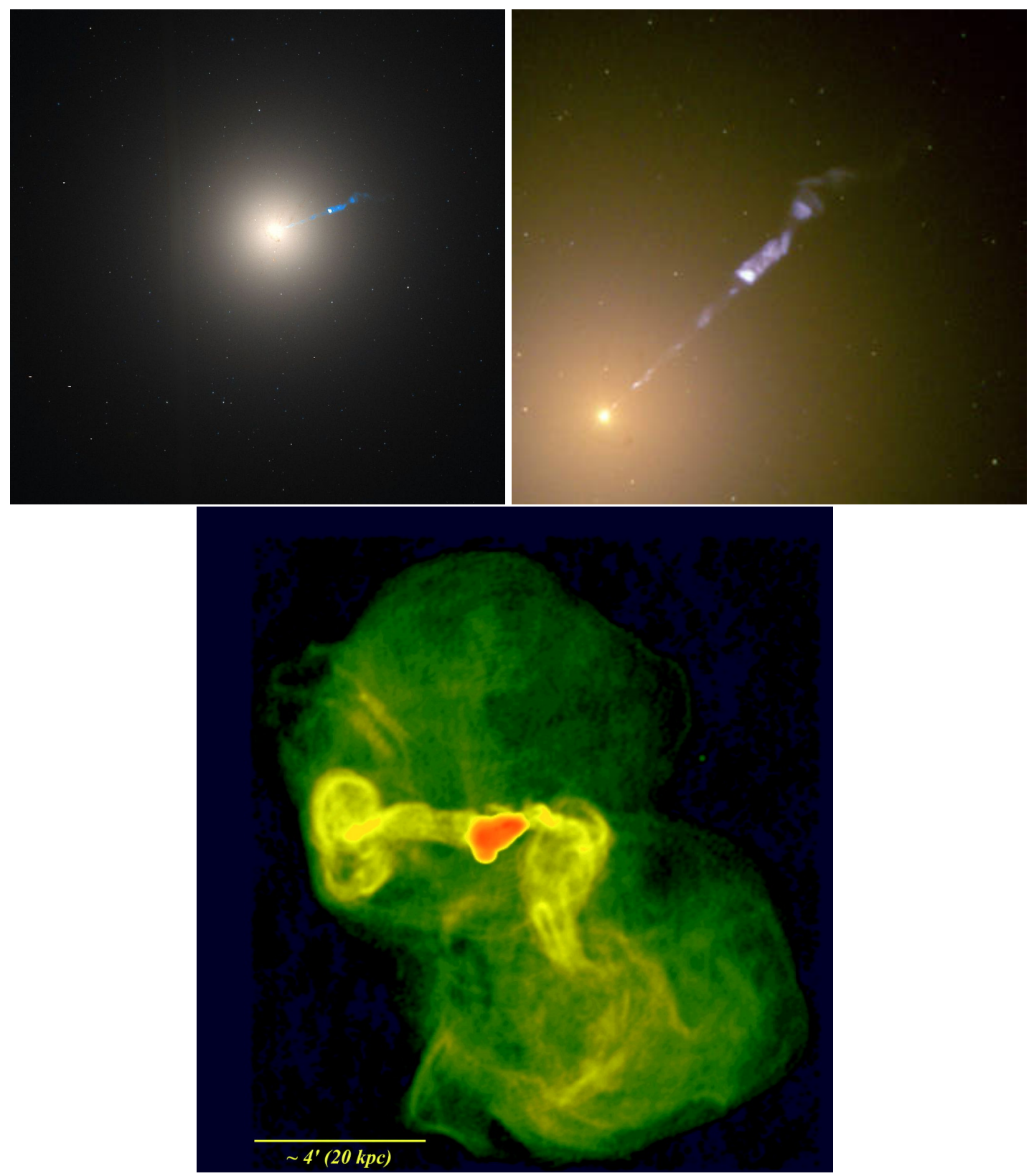

Figure 4: The enormous galaxy M87 has undergone many mergers. Top: Spectacular jets are seen projected outward across the galaxy. These jets of relativistic particles are bad news for life caught in their beams. M87 is the site of the cannibalization of roughly 100 Milky Way type galaxies. Credits: NASA, ESA and the Hubble Heritage Team (STScI/AURA). Bottom: A radio image of M87 at 90-cm, shows a very extended old jet in a different direction than the inner (red) active jet. This directional change is strong evidence for a black hole spin-flip during the merger of supermassive black holes. The impact on complex habitability is worsened by the sweeping out of large volumes as the jets precess in advance of the merger. Credit: Owen et al. [23].

exist beyond the limits of the Super-Galactic Habitable Zone [2] (SGHZ), see Figure 7, where 


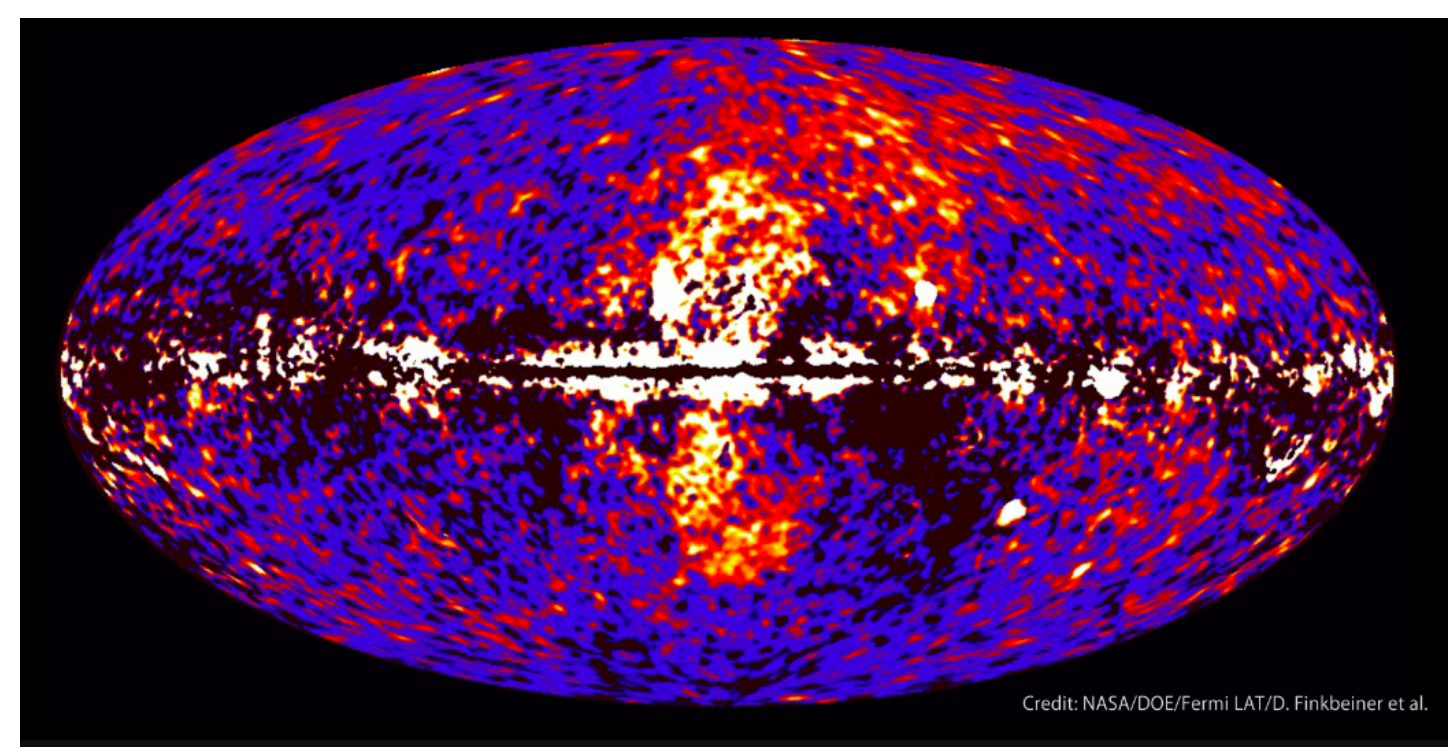

Figure 5: Fermi bubbles of trapped particles are shown as the orange/yellow lobes of high energy gammaray emission above and below the Galactic center. Along the Galactic disk plane, emission from trapped particles giving rise to the Galactic disk wind is also seen. Credits: NASA/DOE/Fermi LAT D. Finkbinder et al.

complex life is highly improbable.

\subsection{Starburst galaxies are detrimental to complex life}

We use as an example the nearby starburst galaxy M82, see Figure 6. The binary black hole merger rate derived by Biermann et al. [20] of $40 t_{3.4}^{-1} G p c^{-3} y r^{-1}$, with 2,500 years $=t_{3.4}$ the estimated time-scale between such mergers in a starburst galaxy such as M82, is now confirmed by LIGO+VIRGO [24, 25] as $56_{-27}^{+44} G p c^{-3} y r^{-1}$. The time scale of repetition of $t_{3.4}=2,500$ years can be checked in two independent ways, i.e. $t_{3.4} \simeq 1$, first via the UHECR flux observed by the Telescope Array Collaboration (TA) [26], and second via the GRB and relativistic SN rate derived by Soderberg et al. [27], and they both agree. So the prediction is consistent. Using this argument in this way implies the assumption that most such black hole mergers involved two black holes of independent (so different) spin-directions will result in a wider detrimental impact to habitability as opposed to a stationary jet. What is currently happening in M82 [20] would be the fate of any galaxy undergoing a major merger, and so every roughly 3,000 years (for a galaxy similar to M82), for probably 3 million years, so roughly 1,000 times (i.e. a stellar mass black hole merger, with the consequences of powerful jets clearing our a large cone, detected in M82 [19], as mentioned. So a fate such as experienced by M82 (currently taking place in the local universe) would probably end civilizations, or force them to repatriate to somewhere else, indeed. Astrophysical knowledge might help, allowing for a long term warning time that would be the length of the starburst, of order $10^{6}$ to $10^{7}$ years; the less informed, short term, warning might be of order 100 years.

Closer to home, the prospects for complex life in the nearby LMC and SMC are not optimistic. These galaxies are metal poor and apparently have had a long history of mutual interaction. They 


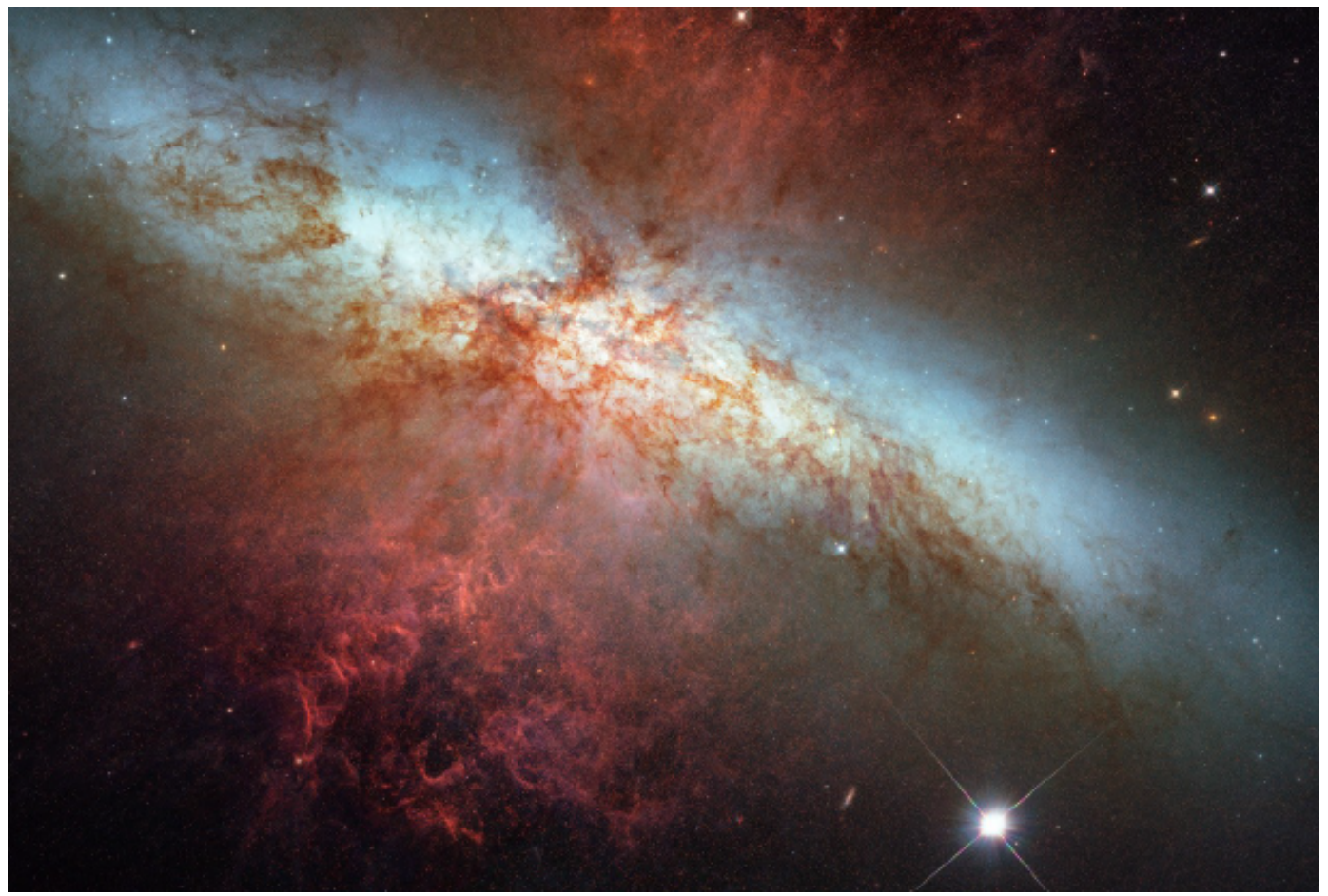

Figure 6: The nearby starburst galaxy M82, The high star formation rate in the central region of M82 produces the galactic wind shown. The environmental conditions within this galaxy likely inhibit the development of complex life. Credit: HST, The Hubble Heritage Team.

are predicted to merge in about 2.5 billion years. Later, in about 4.5 billion years, life in the Milky Way will be compromised. The Andromeda Galaxy and the Milky Way, the two most massive galaxies in the Local Group of galaxies will collide. Three-dimensional space motion of stars in the Milky Way, Andromeda, and the third largest spiral in the Local Group, M33 [28], confirm earlier predictions that theses three will collide resulting in cosmic fireworks, disrupting the Galactic disk and protection for life along with it, and of course the prospect of many stellar mass black holes merging with spin-flips, as now in M82, right along with everything else, like a spike in SNe and GRBs is not optimistic for life.

\section{Challenges for the Far Future}

\subsection{Successful escape comes to DNA selected for intelligence}

Astrobiology, as a science discipline, suffers from having but a single example of life to investigate. In fact, DNA, from an albeit geocentric view, is life on Earth. All life on Earth today is thought to originate from a single Last Universal Common Ancestor (LUCA) [4]. Despite being our only example, life on Earth provides some important clues. The RNA/DNA-based life on Earth today, in all its forms, adapts, through survival and subsequent selection, to a planet that continuously undergoes change. This includes relatively frequent and sometimes catastrophic mass extinctions, pandemics, as well as constant environmental variation. Life has adapted wonderfully 
to predictable environmental change such as seasonal temperature fluctuations and seasonal precipitation. One only needs to look up, to see the migration of birds between higher and lower latitudes in the summer and winter, to see an example; or to herds of mammals on the African savanna following the seasonal rainfall. Life adapts much less readily to stochastic events such as asteroid impacts, volcanic hyperactivity, the removal of atmospheric ozone, due to a supernova and so on. These latter effects result in mass extinctions which are double edged swords for life, cutting off descendancy for entire species, while opening up niches for the survivors.

There are several important points concerning mass extinctions. First, on Earth there does not seem to have ever have been a total extinction, at least since LUCA. If it had happened earlier that that, then we might never know the difference. Life as we know it began early in Earth's history and has never completely disappeared. This is probably of critical importance for the development of complex life on Earth. Life's experiment (on Earth) has not needed to restart in at least the last 3.5 billion years ago or so. However, as has been noted, the survivors on Earth today have done so in spite mass extinction threats and because they have benefited from the mass extinctions of others. Life, especially its development of complex forms, it seems, must remain limited to significant setbacks due to the frequency and severity of mass extinction, by asteroids for example, until an intelligence is developed that is capable of averting such catastrophes. A biosphere subject to heavy or frequent bombardment may stagnate evolutionarily until that frequency decreases. The same is true for any cause of mass extinction. Ultimately, the extinction cycle may terminate only when one group (species), with sufficient intelligence, rises up to protect itself, as well as many other species, from extinction. If that can be achieved, here on Earth or elsewhere by others, then we can say that an advanced level of sustainability has been reached.

Consider that dinosaurs evolved on Earth for hundreds of millions of years, but they could not protect themselves from their ignorance of planetary science. Without destruction by an asteroid, there is no reason to think that things would be much different today. Even greater challenges exist as colossal feats of engineering will be required for members of an apex species to protect themselves. They should attempt to prolong the inevitable end of their planet's habitability. We hope that humans manage to succeed. If humans are able to increase the size of the Earth's orbital distance by adding angular momentum to its orbit, we may avoid global heating due to the slow but inexorable increase in the luminosity of the sun. It is also possible to imagine humans becoming extinct due to a failure to avoid some sort of catastrophe, like pestilence or asteroids. In the long run, in order to survive the nearly endless battle between life and extinction; every conceivable society across the universe must master medicine, astrophysics, planetary protection, and ultimately space colonization. With optimism, we will meet our current challenges. And in the future, the engineering and bioethical challenges that our decedents will certainly face will also be overcome.

Recently, the Earth entered the Anthropocene - the proposed current geological epoch during which human activity is the dominant agent for change to the surface and climate of Earth. Suddenly, and only after 4.5 billion years, Earth now maintains a species, humans, capable of averting global catastrophes. This is an optimistic mark for our civilization, not simply for our species. In this context it is interesting to note that many known great civilizations in the world started by taming flooding. So, the interplay between humans and their environment is as old as civilization itself. Remove 20,000 years of civilization and (large brained) humans are no more capable of planetary protection than a bumble bee. 


\subsection{Colonization poses technological and ethical challenges}

Our intelligent decedents, if humans have them, will face new challenges. They will have to live with themselves among a growing population and limited resources. They will have to deal with otherwise inevitable pandemics, mass delusion, climate change, asteroid and comet impacts, even Earth's long term orbital variations. Eventually, they must address the increase in the luminosity of the Sun as it slowly but inexorably evolves towards becoming a red giant star. If they manage all of that, the merger of the Milky Way with the Andromeda Galaxy may necessitate leaving the Solar System to go to another galaxy for survival. In addition to these technical engineering challenges, astro-bioethical choices must be made. In such cases, not making a choice is in effect a choice, and all such choices have consequences.

To be a successful species into the far future, humans will have learned to deal with all manner of global threats. Including those from its planet, its star, even its galaxy. Colonization of the Moon and Mars appears inevitable. Colonization within and beyond the Solar System will dramatically reduce the risk of human extinction. Consider that a colony of order 100 on a space ship, or a planet, or moon. They could recolonize their home planet a thousand years after an extinction event, like a pandemic or nuclear war, on their planet of origin. The stellar lifetime places a limit on the time needed for a species to rise and then to successfully colonize exoplanets. When Earth's humankind searches for a new planet to settle on, it has to be checked for resident life. The critical question is, what should we value in the resident life we find?

How do we make certain that our value judgements will not go awry, given human history? At first one might be tempted to use the analogy of human evolution, and say that one would differentiate between three cases:

i) We accept the others as equivalent to us and leave them in peace, perhaps open diplomatic relations so as to avoid misunderstandings when our two societies meet elsewhere in the universe.

ii) Deal with them as if they are equivalent to animals and plants, with the most advanced perhaps equivalent to non-human primates, and then confine them to zoos and botanical gardens.

iii) Completely ignore whatever is there.

This last option (iii) may be the only one available if we would get sick or die from some contact. An important lesson comes from Earth colonization. Even if friendly, extraterrestrials may have diseases that we are defenseless against. When two populations meet, and they carry immunity to different diseases, then one of them is victimized, and almost completely wiped out. This happened many times in humanity's history. For example, before Columbus, America probably had more people than all of Europe; about 95\% of them were wiped out by diseases [29]. A biased line of reasoning may be a trap, as the temptation is to consider evolution as a linear progression, whereas the evidence is overwhelming that evolution is highly complex, with many branches, that start suddenly after a considerable change in environmental conditions such as temperature, acidity, water supply and the like. Such branches can end just as suddenly following cosmic catastrophes. If we were to follow, say, option (ii) here, this is equivalent to make the split at that point when chimpanzees separated from the line leading to humans about $5-7$ million years ago. What is the argument to make such an arbitrary split, considering that life on Earth has already lasted more 
than 3 billion years? However, that is the choice that we did make when we consider whom to protect today in zoos and wild parks. On the other hand, our own attitudes have changed considerably within the last hundred years, when slavery was still common, and some people from certain countries were exhibited as 'savages' in exhibitions. Some traces of this survive in politics of today.

However, we could go down another path, and try to communicate with whoever is there, and leave them alone if they do not respond at all, leaving aside the possibility that they may understand our communication, but not care to respond. This option requires a cosmic diplomatic service, a combination of expertise in anthropology, diplomacy, and astrophysics. All this does not even allow for the possibility that we might encounter another society that is considerably more advanced than ours, in terms of space travel, and then likely think about us on their terms. We might also comprehend evolution very much better in the future, and might find that there is a time threshold that defines the minimum time from planet formation to evolve life on its own, say a few billion years, and then only consider planets that are younger than such a threshold. That then leads to the policy that we should mark all such planets as ours already before we go resettle. This in turn leads to seeding such planets with life, but our life, carefully tended, so that whatever evolves there remains friends with us. However, under such a logic, everybody else in the universe might try to do exactly the same. There is no obvious and convincing answer, except to carefully explore whatever and whoever is out there, being careful not to act until consequences are well understood. Considering how much we have learnt in the last 200 years, and how many intellectual revolutions we have gone through, such as with General Relativity and Quantum Mechanics already more than a hundred years ago, and still not fully developed, it is almost certain that we will learn more in the next hundred years that will change our ethical thinking. When considering these complex ethical challenges it will also be incumbent to protect some exoplanet life from exploitation, while accepting inevitable terraforming on other planets.

\section{Conclusions}

Life on Earth has managed to meet countless challenges to get to where we are today. Most of those challenges were met long before our ancestors climbed down from the trees to explore the savanna. Pre-biological chemistry and early organic evolution of replicating molecules, like RNA and DNA, and the formation of the first cells, are important examples. Much later, multicellular life appeared. Relatively recently, plants and animals populated the forbidding land eventually to flourish. Today, we humans seem naturally inclined to peer into the vast expanse of the universe and to wonder, are we alone? This is the essential question of astrobiology, yet attempts to answer it, even state of the art approaches, are subject to a pernicious confirmation bias. Simply put, an enormous multidisciplinary effort has shown that many factors contributed to our existence. However, we are now completely ignorant of the frequency of planets with life, complex or not. That ignorance is merely a current observational limitation. Yet, when combined with the extraordinary hospitable conditions for life on Earth; that bias tends to blind the human mind to the multitude of possible alternative Earth histories.

It is only a matter of luck and apparently only a recent phenomenon that any resident of Earth has been capable of rational thought. Indeed, an argument can be made that present day hominins are only marginally rational beings, since they are only 10,000 generations with brains distinct 
from other hominids. Probably, dolphins or whales have spent substantially more time as the most intelligent species on Earth than humans.

A note on terminology. We say hominin to mean humans and our ancestors until the last common ancestor of chimpanzees. Great apes and hominins, together are hominids. The last common ancestor (LCA) for hominin's lived sometime between 5-7 million years ago. The oldest Homo sapiens are about 300,000 years old, but those specimens did not have modern brain size (though they were close). The LCA date is based on genetics, skeletal evidence, and hypothesis. However, there is no species which anthropologists have designated to be the LCA and there are a lot of arguments about whether certain very early hominin species are actually hominins. There were definitely branching events with all of these species at earlier points of time, chimps most recently, gorillas before that, orangoutangs before that, gibbons before that, monkeys, and so on.

So when we think about 10,000 generations, and count each generation as 25 years, that means 250,000 years. Stone age tools are known from slightly over 3 million years ago. The last common ancestor for hominins and chimpanzees was roughly about 8 million years ago. What, we believe, happened about 400,000 yrs ago, is that we got fire, could cook, and that allowed the brain to grow. Then we began to learn to live together in larger numbers, an on going process, that allowed the human brain to become much more intelligent. [30].

Intelligence is expensive and there is no reason to think that human-like intelligence (defined here as able to escape the planetary nest) is at all inevitable. It is certainly only luck that an asteroid did not destroy nascent humans, delaying the rise of rationalism on Earth. If civilization is destroyed due the our own hand or by external calamity, humanity may recover. If humans are part of a mass extinction, maybe even of all primates, then it will be a challenge for life to develop the next apex species, capable of both superior cognition and technology invention, that will attempt to escape Earth before the end of Earth's habitability, about a billion and a half years from now. An exoplanet may have at best only a few lucky chances to develop beings capable of escaping the end of planetary habitability.

We introduced the concept of a Super-Galactic Habitable Zone in Mason and Biermann (2018) [2] in order to address the question posed in the introduction. Namely, what kind of life will a particular habitable planet support? We suggest that conditions for complex life as we know it are extremely dependent on location and time. Figure 7 shows the Virgo Supercluster, of which the Local group of Galaxies is member. Overlaying this map is a qualitative illustration of the Super-Galactic Habitable Zone. Starburst galaxy phases, supermassive black hole activity, and especially supermassive black hole mergers, occur frequently in rich clusters of galaxies. Rather than excluding the possibility of life in regions dense with galaxies, we argue that the probability for the development of complex life is dramatically reduced over the local value. This is a bit like the Galactic Habitable Zone concept [31], where the dangers in the inner parts of the Milky Way outweigh the benefits of high metallicity and the low metallicity regions in the outskirts of the Galaxy provide for too few concentrated metals. It is significant in this context that planetary protection by a species has taken about $75 \%$ of the habitable lifetime of Earth.

The SGHZ dramatically evolves with time [2]. The elemental abundances slowly enrich the medium out of which stars are formed. The threats to complex surface life decrease by orders of magnitude as the Galaxy has evolved from formation to its current state. Now consider the evolution of the habitable structures within the SGHZ, see Figure 7. In the far future, of order 100 


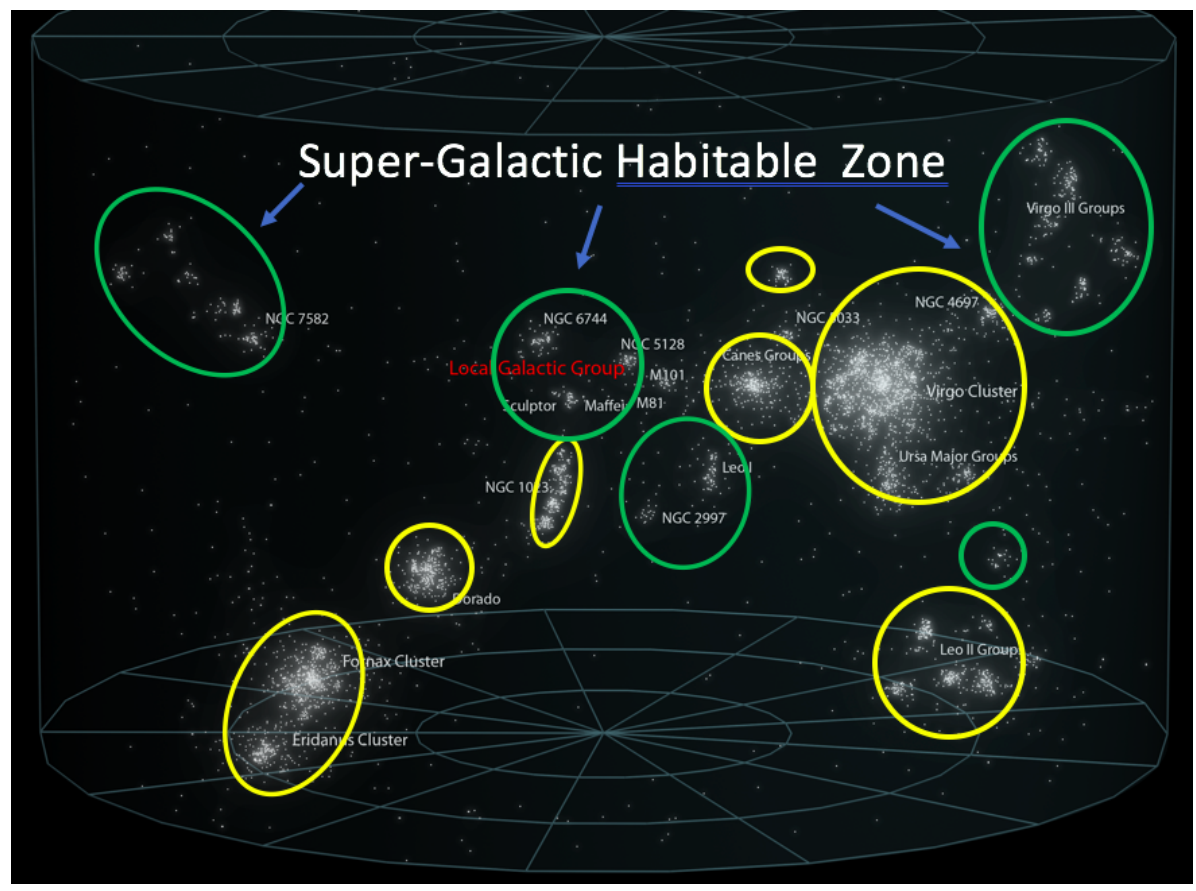

Figure 7: A possible Super-Galactic Habitable Zone (SGHZ). The highest probability for the existence of complex life is likely in regions of intermediate density, within the green bubbles. The higher density galaxy clusters, within the yellow bubbles, are regions of frequent galaxy and black hole mergers. The vast low density (and low metallicity) volume outside of all of the shapes is also beyond the limits of the SGHZ. Credit: Andrew Z. Colvin with adaptation.

Gyr (note: we are not aware of a simulation that goes this far, so $100 \mathrm{Gyr}$ should be considered to be only an order of magnitude estimate), the mega-Galaxy consisting of the merged remains of the Local Group will slowly descend towards the center of the Virgo Supercluster. The new galaxy will be a newly formed region of the SGHZ. Complex life may again thrive, if either it survived the merger event or if it originated afterwards. However, as the Virgo cluster approaches, after 100 Gyr of post-merger calm, they better get out of the way again!

\section{Will humans protect themselves into the unforeseeable future?}

Optimistically, we are on the cusp of that ability at present. The key is to change engage our intellect to overcome our otherwise inevitable demise. The absence of a planet wide catastrophe since the KT impact, 65 million years ago, and significantly, the lack of a snowball Earth period since the Cambrian Explosion 241 million years ago, suggest that good fortune has played an important role in Earth's life. We have now made it to the Antropocene. The key question is now, where do we go from here?

\section{Acknowledgements}

PAM acknowledges travel funding from Picture Rocks Observatory and thanks Franco Giovannelli for the after dinner talk invitation. This paper is derived from several presentations including those given by PLB with the title Principles of Foreign Policy for the next 100 billion 
years, given at Castel Gandolfo, Italy, 5 Sep, 2019, as well the talk given in Mondello (Palermo), Italy, 6 Sep. 2019, with the title of this paper. We especially thank Rebecca Biermann Gürbüz for anthropological discussions that contributed to and improved this paper.

\section{References}

[1] Ward, P. \& Brownlee, D., 2000, Rare Earth, Copernicus press.

[2] Mason, P. A., Biermann, P. L., 2018. Astrophysical and Cosmological Constraints on Life, In: Gordon, R., Sharov, A.A. (Eds.), Habitability of the Universe before Earth. Elsevier B.V, Amsterdam, 89-126.

[3] Dodd, M. S., et al., 2017, Nature. 543, 60.

[4] Theobald, D.L., 2010, Nature. 465, 219.

[5] Biermann, P. L., et al. 2014, MNRAS, 441, 1147.

[6] Johnson, J., 2019, Science, 363, 474.

[7] Mason, P. A., et al. 2013, ApJ Lett., 774, L26.

[8] Mason, P. A., et al. 2015, Astrobiology, 14, 391.

[9] Zuluaga, J., et al. 2016, ApJ, 818, 160.

[10] Peebles P. J. E., 1994, ApJ, 429, 43.

[11] Cox, T. J., Loeb, A., 2008, M.N.R.A.S. 461.

[12] Burbidge, E. M., Burbidge, G. R., Fowler, W. A., Hoyle, F. 1957, Rev. Mod. Phys. $29,547$.

[13] Eichler, D., Livio, M., Piran, T., Schramm, D. N., 1989, Nature 340, 126.

[14] Abbott, B. P. et al., 2017, Phys. Rev. Lett. 119, 161101.

[15] Watson, D., Hansen, C.J., Selsing, J. et al., 2019, Nature 574, 497.

[16] Schrödinger, E., 1944, from lectures given at Trinity College, Dublin.

[17] Gilliland, R. L., et al. 2000, ApJ, 545, L47.

[18] Hanasz, M., et al., 2013, ApJ Lett., 777, L38.

[19] Kronberg, P. P., Biermann, P. L., Schwab, F. R., 1985, ApJ, 291, 693.

[20] Biermann, P. L., et al., 2018, AdSpR, 62, 2773.

[21] Gergely L., \& Biermann, P.L., 2009, ApJ, 697, 1621.

[22] Gopal-Krishna, Biermann, P. L., de Souza, V., Wiita, P. J., 2010, ApJL, 720, L155.

[23] Owen, Eilek, \& Kassim 2000, ApJ, 54,3 611.

[24] Abbott, B. P., et al., 2018, Phys. Rev. Lett. 116, 061102.

[25] Abbott, B. P. et al., 2016, Phys. Rev. Lett. 116, 241103.

[26] Abbasi R. U. et al. [Telescope Array Collaboration], 2015, Astropart. Phys. 64, 49.

[27] Soderberg, A.M., Chakraborti, S., Pignata, G., et al., 2010, Nature, 463, 513.

[28] van den Marel, R. P. et al., 2019, ApJ, 872, 24. 
[29] Lepore, J., 2019, These Truths: A History of the United States. W.W. Norton \& Company

[30] Mason P. A., 2018, The Big-Bang to Big Brains in Frontier Research in Astrophysics - III. 2018. Mondello (Palermo), Italy.

[31] Gonzalez, G., Brownlee, D., Ward, P., Icarus, 152, 185. 